\section{Einladung zum Lebkuchenkongress 2020 DIGITAL}

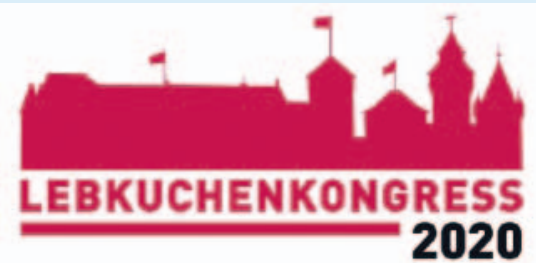

Sehr geehrte Damen und Herren, liebe Kolleginnen und Kollegen,

die äußerst dynamische Entwicklung der COVID-19-Pandemie berührt bereits seit Jahresanfang alle Bereiche des öffentlichen Lebens. Beruflich oder privat, wir alle sind davon in unterschiedlicher Art und Weise betroffen. Vor diesem Hintergrund hat das Programmkomitee in Absprache mit der Deutschen Röntgengesellschaft auf diese völlig neue Situation reagiert und die Umsetzung eines Transformationsprozesses in die Wege geleitet: Aus dem „49. Fortbildungskongress in der Radiologie, Nuklearmedizin und Strahlentherapie - Lebkuchen- kongress 2020“ wird „Lebkuchenkongress 2020 DIGITAL“.

Der 49. Fortbildungskongress in der Radiologie, Nuklearmedizin und Strahlentherapie am 04. und 05. Dezember 2020 in der Meistersingerhalle Nürnberg ist unter den gegebenen Umständen nicht durchführbar und wird in der geplanten Form daher nicht stattfinden. Stattdessen erwartet die Teilnehmerinnen und Teilnehmer zum ursprünglichen Termin am 4. und 5. Dezember 2020 ein modernes digitales Fortbildungsprogramm mit den attraktiven Themenschwerpunkten Thorax, COVID-19 und Bronchialkarzinom.

Wir würden uns freuen, wenn Sie uns auf diesem digitalen Weg begleiten - als Teilnehmerin und Teilnehmer, als Unterstützerin und Unterstützer oder auch nur als wohlwollender Beobachter. Sollten Sie Fragen haben, wenden Sie sich gerne an uns: kongress@drg.de.
Wir wünschen allen Kolleginnen und Kollegen sowie unseren Partnern Gesundheit und viel Kraft, um die auch in den kommenden Herbstmonaten vor uns liegenden Herausforderungen zu meistern.

\section{LEBKUCHENKONGRESS 2020 DIGITAL}

04./05. Dezember 2020

Jetzt anmelden! Alle Informationen und die Anmeldung finden Sie unter: www.lebkuchenkongress.de Wissenschaftliche Leitung:

Prof. Dr. Michael Lell

Katja Röhr

Dr. Josefin Hartmann

Dr. rer. nat. Michael Wucherer

Prof. Dr. med. Dr. rer. nat. Reinhard

Loose

TIPP: Die Fachkundeaktualisierung nach RöV und StrlSchtVer ist komplett online möglich. 